\title{
Oligolactate-Grafted Dextran Hydrogels: Detection of Stereocomplex Crosslinks by X-ray Diffraction
}

\author{
S. J. de Jong, ${ }^{1}$ C. F. van Nostrum, ${ }^{1}$ L. M. J. Kroon-Batenburg, ${ }^{2}$ J. J. Kettenes-van den Bosch, ${ }^{3}$ \\ W. E. Hennink ${ }^{1}$ \\ ${ }^{1}$ Department of Pharmaceutics, Utrecht Institute for Pharmaceutical Sciences (UIPS), Utrecht University, Utrecht, \\ The Netherlands \\ ${ }^{2}$ Department of Crystal and Structural Chemistry, Bijvoet Center for Biomolecular Research, Utrecht University, \\ Utrecht, The Netherlands \\ ${ }^{3}$ Department of Biomolecular Mass Spectrometry, Utrecht Institute for Pharmaceutical Sciences (UIPS) and Bijvoet \\ Center for Biomolecular Research, Utrecht University, Utrecht, The Netherlands
}

Received 2 January 2001; accepted 28 November 2001

\begin{abstract}
Physically crosslinked hydrogels are formed upon mixing equal amounts of aqueous solutions of dextran grafted with L-lactic acid oligomers and dextran grafted with D-lactic acid oligomers. In this paper, the dex-lactate hydrogels are analyzed by X-ray diffraction to provide evidence that hydrogel formation occurred through stereocomplex formation. First, the stereocomplex formation of monodisperse lactic acid oligomers was studied and comparison with published results of poly(lactic acid) was made. It was clearly demonstrated that stereocomplexes were formed be-
\end{abstract}

tween oligomers of opposite chirality. The data for these monodisperse lactic acid oligomers were used to interpret the results obtained with the dex-lactate hydrogels. X-ray diffraction measurements demonstrated that in the hydrogels stereocomplexes were formed between the lactic acid oligomers of opposite chirality. (c) 2002 Wiley Periodicals, Inc. J Appl Polym Sci 86: 289-293, 2002

Key words: stereocomplex; hydrogel; X-ray diffraction; lactic acid oligomer; dextran

\section{INTRODUCTION}

In recent years an increasing number of articles have appeared in the literature on stereocomplex formation in polymer blends. Poly(lactic acid) (PLA) stereocomplexes were studied extensively as a new class of biodegradable materials with higher mechanical strength, improved thermal stability, and less sensitivity to hydrolysis than synthetic polyesters such as poly (glycolic acid) and PLA. ${ }^{1}$ For example, stereocomplex PLA fibers were prepared by spinning from a mixed solution of polymers of opposite chirality, i.e., poly(D-lactic acid) (PDLA) and poly(L-lactic acid) (PLLA), and by dry spinning from a melt of PDLA and PLLA to obtain reinforced materials, which are stronger than PLLA alone. ${ }^{2}$

Stereocomplex formation of PLA was first published by Ikada et al. ${ }^{3}$ Upon mixing, PLLA and PDLA racemic crystallites are formed with a higher melting point $\left(T_{m}\right)$ of $230^{\circ} \mathrm{C}$ than that of a homopolymer of PLA $\left(T_{m}=170^{\circ} \mathrm{C}\right){ }^{3,4}$ The higher melting point is ascribed to a denser packing of the PLA helices in the

Correspondence to: W. E. Hennink (w.e.hennink@pharm. uu.nl).

Journal of Applied Polymer Science, Vol. 86, 289-293 (2002) (C) 2002 Wiley Periodicals, Inc.
1:1 mixture. By X-ray studies, the unit cell of the crystals in the single enantiomeric polymer was found to contain two $10_{3}$-helices. ${ }^{5}$ The unit cell of the stereocomplex contains both a right-handed PLLA and lefthanded PDLA helix, which are packed more densely than the helices in the single enantiomeric polymers. ${ }^{5,6}$ The van der Waals forces between the helices are the driving force for the denser packing of the helices in the stereocomplex. As a result, both PLLA and PDLA form a 3 -helix in the stereocomplex crystal. In the stereocomplex crystal, a PLLA segment and a PDLA segment are packed side by side, with a 1:1 ratio of $\mathrm{L}$ and D monomer units; the segments are packed laterally in parallel fashion.

In a previous article we investigated the minimum degree of polymerization (DP) of lactic acid oligomer that is required to form stereocomplexes. ${ }^{7}$ We demonstrated that a DP of at least 11 is required for crystallization of the single enantiomer of lactic acid oligomer. However, in a blend of (L)- and (D)-lactic acid oligomers crystallization already occurred at DP $\geq 7$, as detected by differential scanning calorimetry (DSC).

This finding was utilized for the design of a novel type of hydrogel in which crosslinking is established by association of enantiomeric lactic acid oligomers grafted to dextran (dex-lactate, Fig. 1). ${ }^{8}$ Rheology ex- 


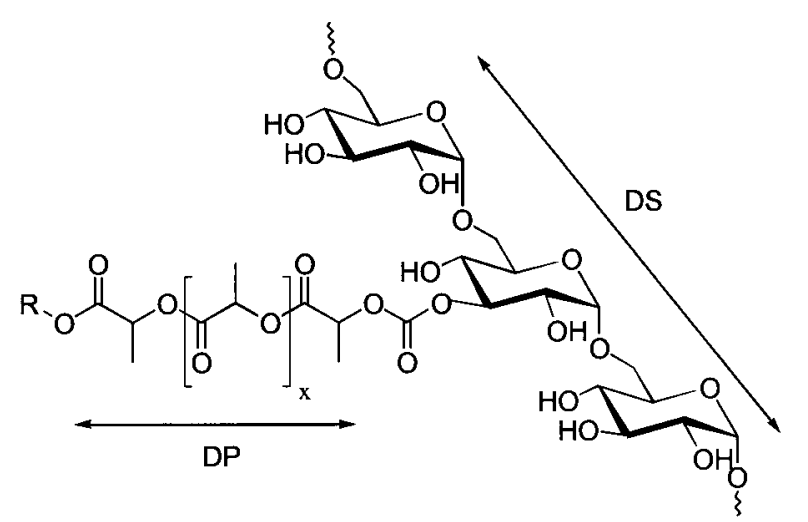

Figure 1 Chemical structure of des-lactate with DP of ( $x$ +2 ) and DS of 1 .

periments showed that a three-dimensional network is formed when two aqueous solutions, containing dex(L)-lactate and dex-(D)-lactate with a DP of at least 11, are mixed. However, although rheology demonstrates that a network is formed, this technique did not provide any information on whether the nature of the association between L- and D-lactic acid oligomers is indeed by stereocomplex formation.

Stereocomplexes can be detected by X-ray diffraction measurement as reported by Lim et al. They showed that stereocomplexes were formed between lactic acid oligomers grafted on poly(2-hydroxyethyl methacrylate) after casting a film from chloroform. ${ }^{9}$ However, any structural evidence of stereocomplex formation in hydrogels has not been provided yet in recent papers suggesting hydrogel formation by stereocomplexation. ${ }^{10-12}$ To gain more conclusive insight into the possible stereocomplex structure in the dexlactate hydrogels, X-ray diffraction analyses on dried products were applied and results are described in this article.

\section{EXPERIMENTAL}

\section{Materials}

L-Lactide [(3S-cis)-3,6-dimethyl-1,4-dioxane-2,5-dione, $>99.5 \%$ ] and ${ }^{\mathrm{D}}$-lactide [(3R-cis)-3,6-dimethyl-1,4-dioxane-2,5-dione, $>99.5 \%$ ] were obtained from Purac Biochem BV (Gorinchem, The Netherlands), and used without further treatment. Stannous octoate [tin(II) bis(2-ethylhexanoate), SnOct $2,95 \%$ ] (Sigma Chemical Co., St. Louis, MO, USA), dichloromethane (Merck, Darmstadt, Germany), and 2(2-methoxyethoxy)ethanol (Aldrich-Chemie, Steinheim, Germany) were used as received. Tetrahydrofuran (THF) and acetonitrile (HPLC-S, gradient grade) were purchased from Biosolve Ltd. (Valkenswaard, The Netherlands). THF was distilled from $\mathrm{LiAlH}_{4}$ immediately before use. Dextran [from Leuconostoc mesenteroides, $M_{n}=15,000 \mathrm{Da}$, and
$M_{w}=32,500 \mathrm{Da}$, as determined by gel permeation chromatography (GPC) analysis] and dimethyl sulfoxide (DMSO, $<0.01 \%$ water) were obtained from Fluka Chemie AG (Buchs, Switzerland). 4-( $N, N$-dimethylamino)pyridine (DMAP, 99\%), and $N, N^{\prime}$-carbonyldiimidazole (CDI, 98\%) were from Acros Chimica (Geel, Belgium). Dialysis tubes [cellulose, molecular weight cutoff 12 000-14 000 (based on proteins)] were purchased from Medicell International Ltd. (London, UK).

\section{Synthesis of (monodisperse) L- and D-lactic acid oligomers}

The lactic acid oligomers were synthesized and purified as previously described by us in detail. ${ }^{7}$ To describe the process in brief: the lactic acid oligomers were synthesized via a ring-opening polymerization reaction of lactide with 2-(2-methoxyethoxy)ethanol (MEE) and stannous octoate as initiator and catalyst, respectively, at $130^{\circ} \mathrm{C}$ for $4 \mathrm{~h}$. The average degree of polymerization $\left(\mathrm{DP}_{\mathrm{av}}\right)$ was tailored by the monomer/ initiator ratio. The monodisperse lactic acid oligomers were obtained by fractionation of polydisperse lactic acid oligomers with preparative HPLC.

\section{Synthesis of dex-(L)-lactate and dex-(D)-lactate}

Polydisperse and monodisperse lactic acid oligomers were coupled to dextran as described in detail by us. ${ }^{7,8}$ After activation of the hydroxyl end group of the lactic acid oligomer with CDI in THF at ambient temperature for $4 \mathrm{~h}$, the resulting lactate-CI was coupled to dextran. The coupling reaction was carried out at ambient temperature for 4 days, with DMSO as solvent and DMAP as catalyst. ${ }^{8}$ The degree of substitution (DS, number of lactate grafts per 100 glucopyranose units of dextran) was calculated from ${ }^{1} \mathrm{H}$ NMR spectra.

\section{Preparation of stereocomplexes and hydrogels}

For the preparation of the oligolactate stereocomplexes, L-lactic acid oligomer and D-lactic acid oligomer of corresponding DP were dissolved in acetonitrile $(20 \mathrm{mg} / \mathrm{mL})$. The solutions were mixed, and the lactic acid oligomer stereocomplex was obtained after acetonitrile was allowed to evaporate. The stereocomplex dex-lactate hydrogel was obtained $24 \mathrm{~h}$ after mixing aqueous solutions of dex-(L)-lactate and dex-(D)lactate $\left(\mathrm{DP}_{\mathrm{av}}\right.$ 12, DS 6, $\left.300 \mathrm{mg} / 700 \mu \mathrm{L}\right)$. Rheology demonstrated that a gel was formed. ${ }^{8}$ The dex-lactate hydrogel was dried overnight at room temperature. 


\section{X-ray diffraction analysis}

X-ray diffraction patterns of all samples were recorded with a Nonius $\kappa$-CCD diffractometer using $\mathrm{MoK} \alpha$ radiation $(\lambda=0.7107 \AA)$ and a graphite monochromator. All patterns were recorded at a sample-to-detector

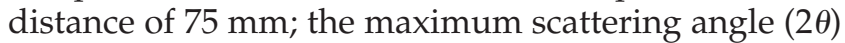
was $22^{\circ}$. Separate blank patterns were recorded to allow subtraction of air scattering. The patterns were integrated at constant $2 \theta$ values so as to obtain radial profiles. Diffraction patterns were recorded for both dextran and dex-lactate, and the diffraction pattern of dextran was subtracted from that of dex-lactate. The spacing $d$ was calculated using the Bragg equation $\lambda$ $=2 d \sin \theta$.

\section{RESULTS AND DISCUSSION}

X-ray diffraction patterns were recorded to detect the presence of stereocomplexes in blends of L- and Dlactic acid oligomers and the dex-lactate hydrogels. Monodisperse lactic acid oligomers were used as a reference to explain the diffraction patterns of the dex-lactate products. Figure 2 shows the X-ray patterns of L-lactic acid oligomer DP 7 and 12 [Fig. 2(A) and $2(\mathrm{C})$, respectively], and the blend of $\mathrm{L}-$ and $\mathrm{D}-$ forms of lactic acid oligomers DP 7 and 12 [Fig. 2(B)

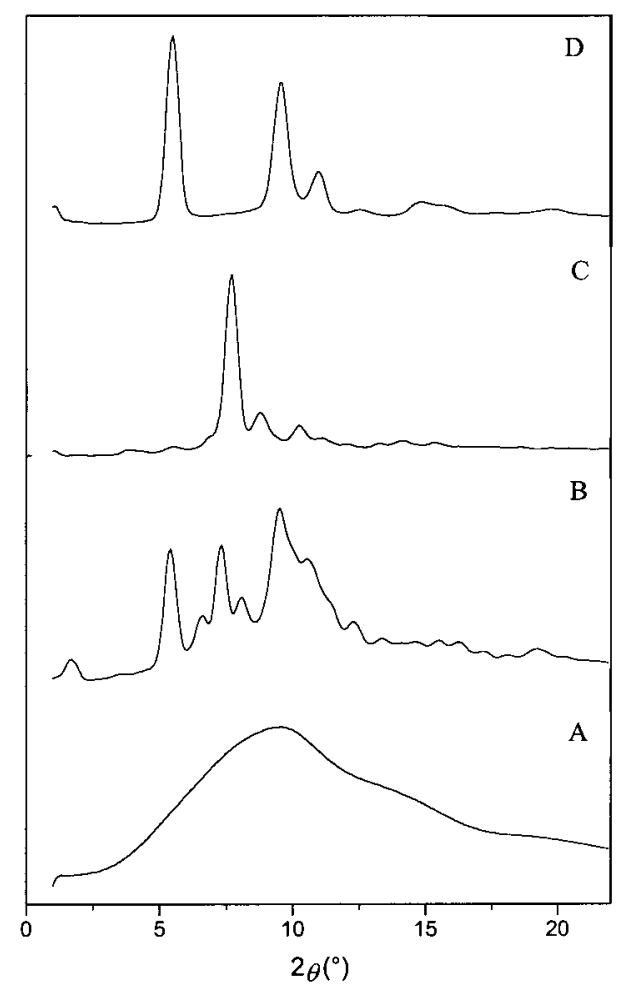

Figure 2 Diffraction patterns of mondisperse L-lactic acid oligomer DP 7 (A), mixture of L- and D-forms DP 7 (B), L-lactic acid DP $12(\mathrm{C})$, and mixture of $\mathrm{L}$ - and D-forms DP 12 (D).
TABLE I

Calculated $d$ Values

\begin{tabular}{|c|c|c|c|c|}
\hline One enantiomeric form & \multicolumn{4}{|c|}{ Calculated $d(\AA)$} \\
\hline HMW PLA homopolymera & 3.99 & & & 5.31 \\
\hline L-Lactate oligomer DP 12 & 3.89 & & & 5.30 \\
\hline L-Lactate oligomer DP 7 & 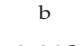 & & & \\
\hline dex-(L)-Lactate DP 12, DS 6 & $3.99^{\mathrm{c}}$ & & & 5.30 \\
\hline Mixture L- and D-forms & \multicolumn{4}{|c|}{ Calculated $d(\AA)$} \\
\hline HMW PLA stereocomplex ${ }^{a}$ & 3.71 & 4.28 & & 7.41 \\
\hline$(\mathrm{L}+\mathrm{D})$-Lactate oligomer DP 12 & 3.71 & 4.26 & & 7.42 \\
\hline$(\mathrm{L}+\mathrm{D})$-Lactate oligomer DP 7 & 3.87 & 4.30 & 5.57 & 7.53 \\
\hline dex-(L+D)-Lactate DP 12, DS 6 & 3.74 & 4.33 & 5.31 & 7.33 \\
\hline
\end{tabular}

\footnotetext{
${ }^{a}$ References 5 and 6.

${ }^{\mathrm{b}}$ No diffraction peaks detected.

c Shoulder.
}

and 2(D), respectively]. The lactic acid oligomer with DP 7 is clearly amorphous, whereas the lactic acid oligomer with DP 12 is highly crystalline. For one enantiomeric form of lactic acid oligomer DP 12, the major diffraction peaks are observed at $2 \theta=7.7^{\circ}, 8.8^{\circ}$, and $10.2^{\circ}$ [Fig. 2(C)]. Table I clearly shows that the corresponding $d$ values are similar to those reported for a high molecular weight (HMW) PLA homopolymer. ${ }^{5,6,9,13}$ Figure 2(D) shows the diffraction pattern of the $\mathrm{L}^{-}$and D-blend of lactic acid oligomer DP $12(2 \theta$ $=5.5^{\circ}, 9.6^{\circ}$, and $10.9^{\circ}$ ), which is identical to that of HMW PLA stereocomplexes (Table I). ${ }^{5,6}$ These results indicate that for the mixture of lactic acid oligomers DP 12 the same $3_{1}$-helical structure is obtained for the stereocomplex as reported for PLA stereocomplexes, and that one enantiomeric form of DP 12 shows a typical PLA homopolymer structure, namely $10_{3}$-helical structure. In agreement with previously reported DSC data, ${ }^{7}$ the one enantiomeric form of lactic acid oligomer DP 7 did not show any crystallinity [Fig. 2(A)], whereas for the corresponding blend of L- and D-forms of DP 7 powder-like crystallinity was detected [Fig. 2(B)]. The stereocomplex of DP 7 forms a three-dimensional crystal structure that is not typical for a fiber structure and therefore has more angle diffraction peaks. Nevertheless, some strong reflections already correspond with the fiber-like DP 12 structure (equator, reflections $h k 0$ ). On the basis of these data, the conclusion that in blends of $\mathrm{L}-$ and D-lactic acid oligomers stereocomplexes are formed is justified.

Because of the limited availability of monodisperse lactic acid oligomers, polydisperse lactate oligomers were coupled to dextran to yield the dex-lactate products used for the X-ray diffraction measurements. Figure 3 shows the X-ray diffraction patterns of dried dextran [Fig. 3(A)], dried dex-(D)-lactate with an average degree of polymerization $\left(\mathrm{DP}_{\mathrm{av}}\right)$ of 12 and a $\mathrm{DS}$ of 6 [Fig. 3(B)], and of the corresponding dried dex- 
lactate gel [mixture of dex-(L)-lactate and dex-(D)-lactate] [Fig. 3(C)]. The rather broad diffraction patterns show that there is little crystallinity in the dex-lactate compounds, which mainly consist of dextran. In both patterns small angle scattering $\left(2 \theta<2^{\circ}\right)$ is observed, which is not detected in the diffraction pattern of pure dextran [Fig. 3(A)]. This indicates that nanodomains are present in both dex-lactate products (single enantiomer as well as the mixture). These domains can most likely be ascribed to association of the hydrophobic lactic acid oligomers. In the diffraction pattern of the mixture of dex-(L)-lactate and dex-(D)-lactate (dexlactate gel) extra peaks (i.e., more structure) are clearly seen when compared with one enantiomeric form of dex-lactate [e.g., peak at 5.6 ${ }^{\circ}$, Fig. 3(C)].

When the diffraction pattern of dextran was subtracted from the patterns of the dex-lactate compounds, substantially more information was obtained (Fig. 4). The intensity of the peaks depended on the amount of dextran signal that was subtracted, but the peak positions were always observed at the same diffraction angles. In the resulting differential diffraction pattern of dex-(D)-lactate [Fig. 4(B)], three peaks were detected at $2 \theta=7.7^{\circ}$ (sharp), at $2 \theta=8.9^{\circ}$ (broad), and at $2 \theta=10.2^{\circ}$ (shoulder), which correspond to the

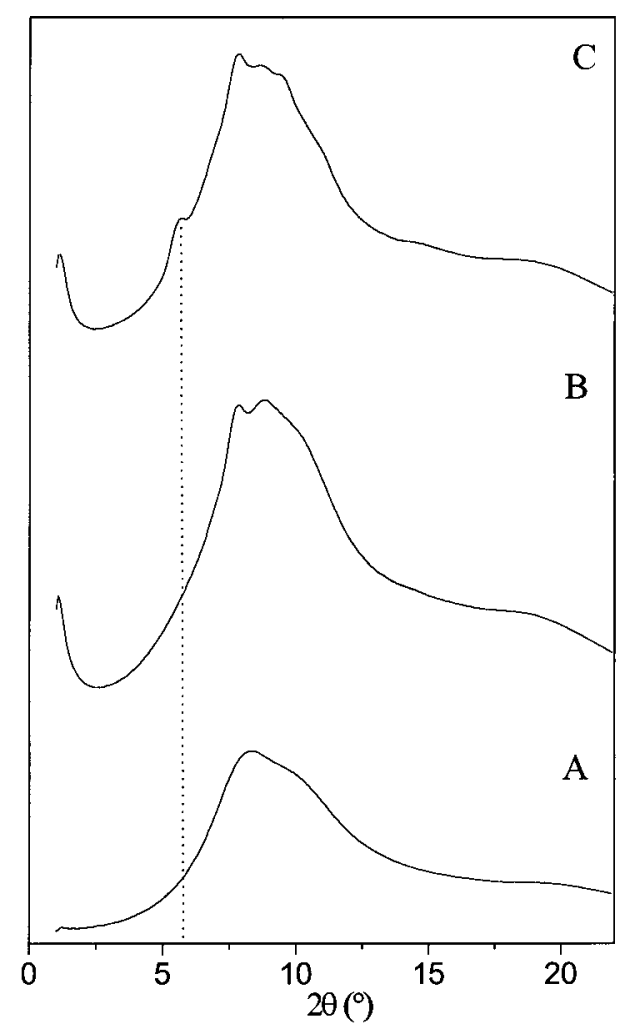

Figure 3 X-ray diffraction patterns of dextran (A); dex-(D)lactate $\mathrm{DP}_{\mathrm{av}} 12$, DS 6 (B); and corresponding mixture of dex-(L)-lactate and des-(D)-lactate (C). The dotted line indicates the extra peak at $5.6^{\circ}$ for the stereocomplex gel.

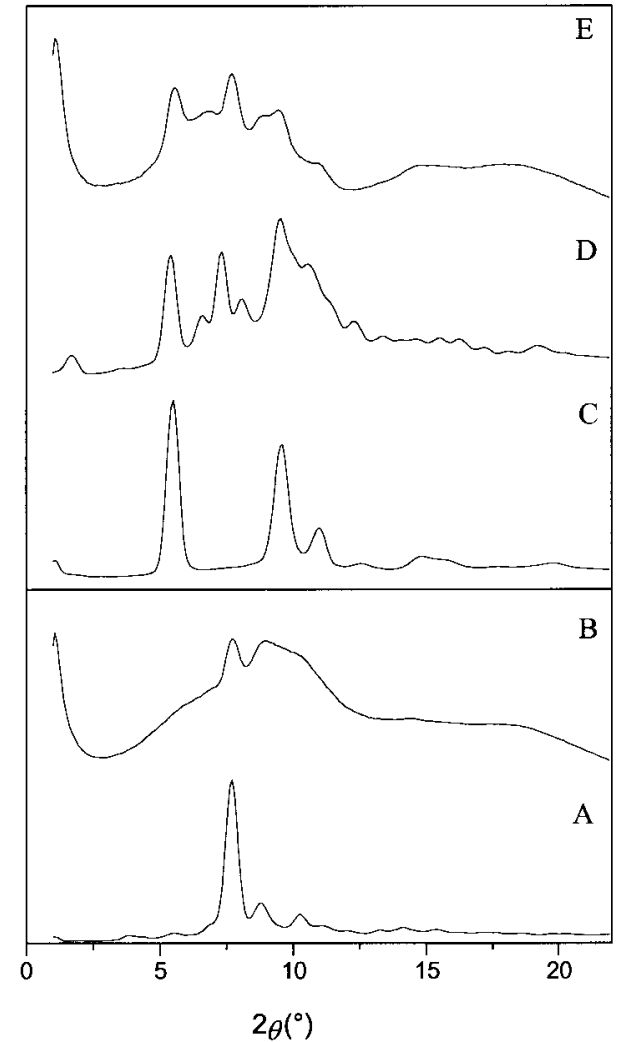

Figure 4 X-ray (differential) diffraction patterns of L-lactic acid oligomer DP 12 (A); dex-(D)-lactate DP ${ }_{\text {av }}$ 12, DS 6 (B); mixture of L- and D-forms of lactic acid oligomer DP 12 (C), lactic acid oligomer DP 7 (D), and dex-lactate DP av 12, DS 6 (E) (the diffraction patterns of the oligomers are from Fig. 1 for comparison).

peaks observed for crystalline (L)-lactic acid oligomer DP 12 [Fig. 4(A)]. The dex-lactate L- and D-mixture [Fig. 4(E)] shows several diffraction peaks that correspond to those of the lactic acid oligomer mixtures [Figs. 4(C) and 4(D) and Table I)], indicative of the presence of stereocomplex.

\section{CONCLUSIONS}

From the X-ray data it can be concluded that the lactic acid oligomers grafted to dextran are responsible for crystallinity in both the dex-(D)-lactate product and the dex-lactate gel. In the dex-(D)-lactate the crystallinity is due to normal oligolactate (DP $\geq 11$ ) crystallization, whereas in the dex-lactate gel it was clearly demonstrated that stereocomplex crystals are formed after mixing aqueous solutions of dex-(L)-lactate and dex-(D)-lactate.

\section{References}

1. Tsuji, H.; Ikada, Y. Polymer 1999, 40, 6699-6708.

2. Tsuji, H.; Ikada, Y.; Hyon, S.; Kimura, Y.; Kitao, T. J Appl Polym Sci 1994, 51, 337-344. 
3. Ikada, Y.; Jamshidi, K.; Tsuji, H.; Hyon, S.-H. Macromolecules 1987, 20, 904-906.

4. Tsuji, H.; Hyon, S.-H.; Ikada, Y. Macromolecules 1991, 24, 56575662.

5. Okihara, T.; Tsuji, M.; Kawaguchi, A.; Katayama, K. J Macromol Sci, Phys 1991, B30, 119-140.

6. Brizzolara, D.; Cantow, H.-J.; Diederichs, K.; Keller, E.; Domb, A. Macromolecules 1996, 29, 191-197.

7. de Jong, S. J.; van Dijk-Wolthuis, W. N. E.; Kettenes-van den Bosch, J. J.; Schuyl, P. J. W.; Hennink, W. E. Macromolecules 1998, 31, 6397-6402.
8. de Jong, S. J. De Smedt, ; S. C.; Wahls, M. W. C.; Demeester, J.; Kettenes-van den Bosch, J. J.; Hennink, W. E. Macromolecules 2000, 33, 3680-3686.

9. Lim, D. W.; Choi, S. H.; Park, T. G. Macromol Rapid Commun 2000, 21, 464-471.

10. Lim, D. W.; Park, T. G. J Appl Polym Sci 2000, 75, 1615-1623.

11. Grijpma, D. W.; Feijen, J. Proc 6th Eur Symp Controlled Drug Delivery 2000, 113-115.

12. Fujiwara, T.; Mukose, T.; Yamaoka, T.; Yamane, H.; Sakurai, S.; Kimura, Y. Macromol Biosci 2001, 1, 204-208.

13. Li, S.; Vert, M. Polym Int 1994, 33, 37-41. 